\title{
TIPOLOGÍA DEL ORDEN DE PALABRAS EN BRIBRI
}

\author{
Carla Victoria Jara
}

\begin{abstract}
RESUMEN
Este artículo presenta una caracterización de la tipología del orden de palabras en bribri (lengua de la familia chibcha) de acuerdo con algunos parámetros propuestos por Greenberg y Vennemann: el orden de palabras en la cláusula simple, la frase genitiva, las adposiciones, sustantivo-adjetivo, la cláusula relativa, verbo-adverbio, verbo-auxiliar y verbo-modal.
\end{abstract}

\section{ABSTRACT}

This article characterizes the tipology of the word order in Bribri (Chibchan language) according to the following parameters proposed by Greenberg and Vennemann: Word order of the simple clause, genetive phrase, adpositions, noun-adjective, relative clause, verb-adverb, verb-auxiliar and verbmodal particles.

\section{Introducción}

\subsection{Antecedentes generales e históricos de los bribris}

Existen en la actualidad seis grupos indígenas en el territorio costarricense: malekus (también llamados guatusos), bribris, cabécares, térrabas, borucas y guaymíes ${ }^{1}$. Todos estos grupos hablan, o hablaron en el caso de los térrabas y los borucas, lenguas de la familia chibcha y se ubican en la región cultural denominada Área Intermedia (para una caracterización de esta, ver Constenla Umaña 1991:7), la cual se extiende, en términos generales, desde la costa atlántica de Honduras hasta el centro de Colombia, e incluye además la parte noroeste de Venezuela y la mitad occidental de Ecuador.

Muchas de las características culturales del área se encuentran en la historia de los bribris: el maíz fue su principal medio de subsistencia, las instituciones sociopolíticas eran reducidas, tenían centros ceremoniales y practicaban sus propias ceremonias fúnebres, etc ${ }^{2}$.

Las referencias históricas acerca de la vida y las costumbres de los nativos de Costa Rica son pocas e imprecisas. Del período colonial, quedan algunas informaciones recogidas por misioneros católicos; de fines del siglo XIX, las principales referencias son Gabb (1875) y Pittier (1898, 1903), y del siglo XX, Stone (1961), Bozzoli (1975a, 1979) y Guevara y Chacón (1992). 
Cultural y lingüísticamente, los pueblos que habitaron Costa Rica se dividen en dos grupos: los pueblos del Pacífico Norte (en el golfo de Nicoya, la península de Nicoya, el valle del Tempisque y el norte de Guanacaste) son de origen mesoamericano, mientras que los grupos del resto del país (planicies de Alajuela, las regiones atlántica y central y el Pacífico Sur) son de filiación chibcha.

Entre los grupos de filiación chibcha, los bribris y los cabécares sobrevivieron el período colonial en su lugar de origen: el valle de Talamanca ${ }^{3}$. Bozzoli (1979: 38) ha señalado que los bribris lograron mantenerse independientes de los europeos gracias a que no permitieron misiones religiosas ni otros asentamientos no nativos en sus dominios hasta 1882, cuando las actividades bananeras de la Chiriqui Land Company invadieron la región talamanqueña.

Como ejemplo de la manera bravía en que los talamanqueños lograron defender su libertad, Guevara y Chacón (1992: 37) cuentan:

\footnotetext{
Las esporádicas incursiones del sistema militar colonial no lograron legitimar posesión alguna por parte de los españoles, ya que generalmente las tierras invadidas fueron posteriormente abandonadas a causa de la imposibilidad de la gobernación por proveer los pueblos fundados de lo necesario, y en razón de la hostilidad de las comunidades indias vecinas, que para resistir los intentos de dominación organizaron ataques constantes, emboscadas, y hasta se dedicaron a quemar sus propios cultivos y reservas alimenticias para impedir que los españoles se abastecieran. Tampoco fueron significativos los intentos de "dominación espiritual" de los misioneros que lograron permanecer en Talamanca hasta principios del siglo XVIII, pues fueron en fin de cuentas expulsados por los indios, cuando no muertos.
}

De acuerdo con Guevara y Chacón (ibid.: 43), en el siglo XX la Chiriqui Land Company dominó Talamanca durante treinta años, después de los cuales la abandonó debido a la pérdida de fertilidad de los suelos, el advenimiento de enfermedades del banano y los daños en la infraestructura causados por las inundaciones. Los talamanqueños así tuvieron la oportunidad de recuperar sus territorios, aunque sus tierras habían sido drásticamente dañadas.

A pesar del constante asedio espiritual, cultural y económico, los talamanqueños lograron preservar algunos aspectos de su vida tradicional, tales como las chichadas, fiestas en que la bebida de chicha es el evento central; formas específicas de cultivar las cosechas tales como las parcelas; patrones complejos en los conceptos de nacimiento, enfermedad y muerte, y una buena cantidad de su tradición oral (Jara y García, en prensa).

\subsection{Los bribris en la actualidad}

Los bribris -cerca de 13000- viven en el sur de Costa Rica, en particular a ambos lados de la cordillera de Talamanca. Algunos grupos de bribris emigraron hacia el lado pacífico de la cordillera y se asentaron en el cantón de Buenos Aires, provincia de Puntarenas, en donde se encuentran las reservas de Salitre y Cabagra. Este grupo lo constituyen aproximadamente 3000 personas, que viven del cultivo de arroz, maíz, frijoles, pejibaye (Guilielma utilis), etc.

Del lado atlántico de la cordillera, los bribris, alrededor de 10000, se concentran en la Reserva de Talamanca, en el cantón de Talamanca, provincia de Limón. Además de los productos ya mencionados del lado pacífico, cultivan cacao y plátanos, que constituyen sus principales productos.

Bozzoli (1979: 56) se refiere a la vida actual de los bribris así: 


\begin{abstract}
Hoy día los bribris, como todos los otros aborígenes costarricenses, se organizan del mismo modo que el resto de la población nacional, en asuntos legales, políticos y administrativos. Las escuelas, los servicios de salud, de policía, de obras públicas, se organizan a nivel nacional. (...) Muy pocas familias han poseído escrituras de sus tierras; mantienen una lucha contra la población nacional en expansión que se apodera de sus predios y bosques.
\end{abstract}

En la actualidad, la mayoría de los bribris lucha por mantener su cultura viva, pero esta lucha no se da solo frente a la cultura dominante sino también entre ellos mismos: entre los que preferirían mantener su forma de vida tradicional intacta y los que querrían asimilarse completamente al resto de la población nacional. Esta falta de acuerdo entre los mismos bribris hace todavía más difícil para ellos defender sus derechos sobre la tierra, la cultura y la educación.

\title{
1.3. La lengua bribri
}

La familia chibcha fue establecida sobre la base del método lexicoestadístico por Constenla Umaña (1985). De acuerdo con este estudio, las lenguas que conforman la familia son el muisca (o chibcha propiamente, de Colombia), el cuna, el movere y el bocotá (de Panamá), el boruca, el térraba y el guatuso (de Costa Rica), junto con los grupos arauaco (cágaba y bíntucua, de Colombia) y viceíta (bribri y cabécar, de Costa Rica). Más tarde, Constenla Umaña $(1989,1991)$ deja la denominación "familia" y adopta el término estirpe chibcha, el cual incluye, además de las lenguas ya mencionadas, el paya (de Honduras), el rama (de Nicaragua), el dorasque y el chánguena (de Panamá), el chimila, el guamaca, el atanques, el bari, el tunebo y el duit (de Colombia).

El bribri carece de un sistema de escritura propio. Para dotar a la lengua de forma escrita, el departamento de linguiística de la Universidad de Costa Rica ha diseñado un "alfabeto práctico", que ha sido utilizado por el Ministerio de Eduación en materiales didácticos para esta lengua. El mismo sistema se ha usado en la mayoría, sino en toda, la investigación reciente sobre la lengua bribri. Los siguientes son los símbolos gráficos correspondientes a los sonidos del bribri y, entre paréntesis, sus equivalentes en Alfabeto Fonético Internacional (IPA):

\section{Vocales}

$\begin{array}{lll} & \text { Orales } & \text { Nasales } \\ \text { Altas: } & \mathrm{i}, \mathrm{u} & \underline{\mathrm{i}}(\tilde{}), \underline{\mathrm{u}}(\tilde{\mathrm{u}}) \\ \text { Medias altas: } & \ddot{\mathrm{e}}(\mathrm{I}), \ddot{\mathrm{o}}(\mathrm{v}) & \\ \text { Medias bajas: } & \mathrm{e}(\varepsilon), \text { o (o) } & \underline{\mathrm{e}}(\tilde{\varepsilon}), \mathrm{o}(\tilde{\mathrm{o}}) \\ \text { Bajas: } & \mathrm{a} & \underline{\mathrm{a}}(\tilde{\mathrm{a}})\end{array}$

\section{Consonantes}

$\begin{array}{ll}\text { Oclusivas } & \mathrm{p}, \mathrm{b}, \mathrm{t}, \mathrm{d}, \mathrm{k}, \mathrm{C}^{\prime}(\mathrm{l}) \\ \text { Nasales } & \mathrm{m}, \mathrm{n}, \tilde{\mathrm{n}}(\mathrm{p}) \\ \text { Africadas } & \mathrm{ts}, \mathrm{ch}(\mathrm{t}), \mathrm{y}(\mathrm{d} 3) \\ \text { Fricativas } & \mathrm{s}, \mathrm{sh}\left(\mathrm{\int}\right), \mathrm{j}(\mathrm{h}) \\ \text { Vibrante simple } & \mathrm{r}(\mathrm{r})\end{array}$




$\begin{array}{ll}\text { Vibrante múltiple } & \mathrm{rr}(\mathrm{r}) \\ \text { Lateral retrofleja } & \mathrm{l}(\mathrm{l}) \\ \text { Oclusiva coarticulada: } & \mathrm{tk}(\mathrm{tch}(\mathrm{tt} \mathrm{f}) \text { en el dialecto de Coroma) } \\ \text { Transiciones: } & \mathrm{w}, \mathrm{i}(\mathrm{j}) \\ \text { Tonos: } & / \text { / } \text { descendente, } / \text { / alto, tono bajo: no marcado. }\end{array}$

En cuanto a su tipología estructural, como se verá, el bribri es una lengua posposicional con marcación de casos absolutivo-verbo, siendo la posición del absolutivo obligatoriamente preverbal. En la cláusula transitiva, la FN ergativa se marca mediante la posposición tö (variantes dör, di, re, r), y puede aparecer tanto delante de la FN absolutiva como después del verbo. Ejemplos:

\section{Cláusula transitiva:}

(1a) Alà tö chạụ ñè̀ke. niño ERG banano comer.IM

(1b) Chamù ñèke alà tö. banano comer.IM niño ERG

'El niño come banano.'

Cláusula intransitiva:

(2) Alà inùke.

niño jugar.IM

'El niño juega.'

\subsection{Consideraciones dialectológicas}

La situación dialectal del bribri no ha sido completamente determinada. Schlabach (1974) reconoce dos dialectos en la región atlántica: Lari (que incluye las comunidades de Lari, Amubre, Urén y Yorkín) y Coén (que incluye Coén, Coroma, Sepecue y Chiroles), y reconoce la posible existencia de un tercer dialecto, el de Salitre, en la región pacífica. Wilson (1982) y Margery (1982) reconocen dos dialectos bien definidos: uno en la región atlántica llamado Amubre (que incluye las comunidades de Amubre y Katsi), y otro en la región pacífica, el de Salitre (que incluye las comunidades de Salitre y Cabagra), y señalan la posible existencia de un tercer dialecto en el lado atlántico, el de Coroma (que correspondería al que Schlabach reconoce como Coén). Sobre la base de diferencias fonológicas, Amubre parece ser el dialecto más innovador, mientras que Coroma representa la variante conservadora ${ }^{4}$. Los datos que se presentan en este artículo corresponden al dialecto de Coroma.

El hecho de que Amubre y Coroma, los dos dialectos talamanqueños, sean los más divergentes entre sí resulta interesante, ya que geográficamente se encuentran más cercanos 
entre sí que cualquiera de los dos con respecto a Salitre. Existe evidencia histórica que claramente explica esta división entre los tres dialectos.

En el caso de los dialectos de Talamanca, Bozzoli (1975b: 31-2) establece la razón histórica sobre la base de la organización clánica y en particular el sistema de matrimonio:

\footnotetext{
The [Bribri] clans were originally distributed along the four main river valleys. In the past the Lari River males married the women in the Uren River Valley, while these women's brothers had to marry their brothers-in-law's sisters in the Lari River Valley. The Coen River Valley clans exchanged marriage partners with the clans located between West Coen and East Telire River Valley. This is probably one of the reasons why the Coen Bribris speak a Bribri dialect which is closer than other Bribri dialects to the Cabecar dialect spoken in their midst and also between West Coen and East Telire, and why Coen clans are reported sometimes as Cabecar, and sometimes as Bribri clan.
}

Hay también razones históricas que apoyan la configuración de un nuevo dialecto en la región pacífica. Como señala Stone (1961: 18):

\begin{abstract}
Los dos grupos bribris [en el Pacífico] se encuentran en Cabagra y en Salitre... La fecha exacta de la llegada de los Bribris a este lugar no se conoce, aunque probablemente fue al final del siglo XIX, puesto que muchos de los más viejos habitantes nacieron cerca de los ríos Lari y Uren. Una tradición sostiene que ciertas familias huyeron a través de la cordillera de Talamanca porque no se habían adherido a regulaciones de clan con respecto a matrimonios. Tal vez sea significativo el hecho de que los grupos en ambos lugares están formados por muy pocos clanes y que como pueblo son los primeros en apartarse de las antiguas costumbres tribales.
\end{abstract}

\title{
2. Tipología del orden de palabras en bribri
}

\subsection{Preliminares acerca del orden de palabras (OP)}

En un artículo pionero sobre la tipología lingüística de OP, Joseph Greenberg (1966) señalaba la noción ya para entonces conocida de que las lenguas tienden a una armonía en el OP según la cual los elementos modificadores o limitantes siguen a los elementos modificados o limitados y viceversa. Sobre la base de datos dialingüísticos, Greenberg (1966: 76) encontró que muchos de los fenómenos relativos al OP reflejan que "algunos factores se relacionan íntimamente mientras que otros son relativamente independientes". Los parámetros que se mostraron relevantes para establecer esta conclusión son los siguientes:

1. Orden básico entre sujeto, verbo y objeto directo en la cláusula simple. Este parámetro permite seis posibles combinaciones; sin embargo, se halló, en una amplia muestra de lenguas, que los órdenes más frecuentes son SOV, SVO y VSO.

2. Orden entre la adposición y su objeto: si el orden es adposición-sustantivo, la lengua es llamada preposicional; si el orden es sustantivo-adposición, la lengua es llamada posposicional.

3. Orden entre el poseedor (genitivo) y el objeto poseído (sustantivo): las opciones son genitivo-sustantivo o sustantivo-genitivo. 
4. Orden entre el sustantivo y el adjetivo: las opciones son adjetivo-sustantivo o sustantivo-adjetivo.

Sobre la base de estos cuatro parámetros, es posible distinguir 24 tipos de lenguas. Greenberg comparó una muestra de 30 lenguas en relación con estos parámetros y encontró ejemplos de 15 tipos. Estos tipos, a su vez, fueron reagrupados en cuatro tipos generales:

a) VSO, preposicional, sustantivo-genitivo, sustantivo-adjetivo;

b) SVO, preposicional, sustantivo-genitivo, sustantivo-adjetivo;

c) SOV, posposicional, genitivo-sustantivo, adjetivo-sutantivo; y

d) SOV, posposicional, genitivo-sustantivo, sustantivo-adjetivo.

Los parámetros para la clasificación tipológica de las lenguas fueron propuestos por Greenberg de manera relativamente independiente unos de otros. Sin embargo, posteriormente se propusieron generalizaciones que apuntaban a correlaciones holísticas entre parámetros. Vennemann (1974), por ejemplo, propuso que el OP tendía a un modelo consistente en que el orden es Adjunto-Núcleo o Núcleo-Adjunto ${ }^{5}$. La siguiente lista (en Vennemann, op.cit.: 345) muestra los elementos correspondientes a cada una de estas categorías:

$\begin{array}{ll}\begin{array}{l}\text { Adjunto } \\ \text { objeto }\end{array} & \text { Núcleo } \\ \text { verbo } \\ \text { adverbio } & \text { verbo } \\ \text { verbo principal } & \text { verbo auxiliar } \\ \text { verbo principal } & \text { verbo modal } \\ \text { modificador } & \text { sustantivo } \\ \text { (adjetivo, } & \\ \text { cláusula relativa, } & \\ \text { atributo adverbial, } & \\ \text { atributo genitivo) } & \\ \text { término de comparación } & \\ \text { frase nominal } & \text { adjetivo comparativo }\end{array}$

Este modelo ha sido criticado (Comrie 1989) porque, a pesar de ser simple y elegante, se toma demasiadas libertades con respecto a los datos disponibles, dejando de lado múltiples excepciones sin justificación. Otro conjunto de universales de OP fue propuesto por Hawkings (1983), pero de nuevo, las excesivas excepciones demuestran que los universales de OP solo pueden proponerse como estadísticos (Campbell et al 1988).

En suma, las tipologías holísticas en cuanto a OP son idealistas debido a múltiples razones, en particular las siguientes: los frecuentes cambios en OP en aras de codificar diferentes significados (determinaciones pragmáticas), la influencia del contacto con otras lenguas (por ejemplo, el préstamo sintáctico, Campbell et al. ibid) y la fuerza del cambio diacrónico (Givon 1984). Sin embargo, una tipología general es sin duda útil para la caracterización de cualquier lengua. Este artículo tiene como objetivo central caracterizar la lengua bribri en este respecto. 


\subsection{OP de la cláusula simple en bribri}

El bribri es una lengua OV con sistema de marcación de casos absolutivo-ergativo. Como tal, es preferible caracterizarlo como una lengua de absolutivo-verbo con OP rígido. El ergativo puede aparecer antes o después de la secuencia absolutivo-verbo: erg-abs-V o abs-V-erg. En el discurso, el sujeto ergativo y en menor grado el sujeto absolutivo (intransitivo) con referente previamente mencionado (es decir, sujetos de segundo plano) son frecuentemente elididos.

Véase el siguiente fragmento del texto que se incluye como apéndice, Tcho'dawe 'Los diablos del bosque' (cláusulas 36-39 reproducidas aquí como 3):

(3a) Kó e' nạnéwe tạ, ie' wạ kapá taì táwa. tiempo DEM noche PROG $3 \mathrm{~S}$ AG jefe grande matar.PE Esa noche él decidió matar a un gran jefe.

(3b) Kalöwewa agarrar.IM

Lo agarró,

(3c) ttèlur,

matar.IM

lo mató

(3d) pàterèwa bua'ë kòkekuö wa.

envolver.IM bien.INT corteza INST

y lo envolvió muy bien con corteza de mastate.

Sin embargo, de las 32 cláusulas simples transitivas ${ }^{6}$ en el texto Tcho'dawe, 24 presentan absolutivo explícito, mientras que solo 8 presentan anáfora cero. Esto muestra la preferencia de la lengua por mantener el absolutivo explícito en el discurso.

En cuanto a la aparición de sujetos ergativos, se encontraron 11 ergativos prepuestos (erg-abs-V), 10 ergativos pospuestos (abs-V-erg) y 11 ergativos elididos (anáfora cero), lo cual muestra la flexibilidad de la lengua en relación con las FNs ergativas. Considérense los siguientes ejemplos:

Cláusula 1:

(4) E' képata Sibö tö îyök yawè,

DEM después dios ERG tierra hacer.IM

Después de que Sibö(dios) hizo la tierra,

Período condicional (cláusulas 23-24):

(5) $\mathrm{Be}^{\prime} \mathrm{r} \quad \mathrm{e}^{\prime}$ mèat,

2S ERG DEM dejar.IM

Si los dejas, 
(6) be' ttèwa i di. 2S matar.IM 3S ERG ellos te matarán.

Agente no típico (cláusula 65):

(7) Tcho'dawe kalöwe di' tö, Tcho'dawe agarrar.IM agua ERG

El agua agarró a Tcho'dawe,

La posición de la FN ergativa, sea delante o después de abs-V, parece estar determinada por la organización del discurso. Quiero sugerir que cuando el participante codificado como ergativo es más prominente/importante/relevante en el discurso en comparación con el participante codificado como absolutivo, aquel tiende a ser preverbal; en caso contrario, tiende a ser posverbal.

Las cláusulas intransitivas también fueron examinadas y aunque sus sujetos absolutivos pueden ser explícitos o no (dependiendo de si fueron mencionados previamente), cuando son explícitos son siempre preverbales, lo cual confirma que la mejor caracterización del OP de la cláusula simple, integrando tanto las cláusulas transitivas como las intransitivas, es abs-V.

Ya hemos señalado que la posición del sujeto ergativo en la cláusula se define pragmáticamente. Por otra parte, este aparece por lo general marcado morfológicamente. De las 21 FNs ergativas explícitas en el texto, 18 están morfológicamente marcadas, la mayoría de ellas con la posposición tö (o sus variantes). Tö también puede contraerse con formas pronominales (por ejemplo yö $=y e^{\prime}$ '1 persona singular' + tö 'marcador de ergativo', ver ejemplo (9) infra y la cláusula DC15). En casos particulares, las FNs agentivas se marcan con la posposición $w \underline{a}$, como en la cláusula 36 (ver 3a supra). En la mayoría de los casos, sin embargo, wa marca el agente de un verbo que es intrínsecamente intransitivo (verbos con objeto locativo y verbos de voz media) cuando son usados como transitivos:

$$
\begin{array}{llcccl}
\text { Ák } & \mathrm{e}^{\prime} & \text { mìne } & \text { ie' } & \text { wa } & \text { à̀. } \\
\text { piedra } & \text { DEM } & \text { ir.PE } & 3 \mathrm{~S} & \mathrm{AG} & \text { allá-arriba } \\
\text { Él llevó la piedra allá arriba. } & &
\end{array}
$$

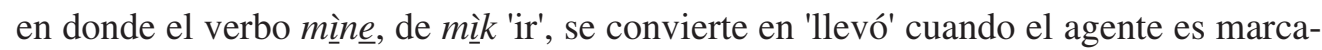
do con wa.

Los casos oblicuos tienden a ser periféricos a abs-V. De acuerdo con mis datos, el dativo es el único caso que puede aparecer entre erg y abs:

$\begin{array}{llllll}\text { Ewa } & \text { yö } & \text { mi } & \underline{a} & \text { i } & \text { pàke. } \\ \text { más-tarde } & 1 S-E R G & 2 S & \text { DAT } & 3 \mathrm{~S} & \text { decir.IM } \\ \text { Te lo diré más tarde. } & & & & \end{array}$




\subsection{Frase genitiva}

El orden de la frase genitiva en bribri es siempre poseedor-poseído (genitivo-sustantivo):

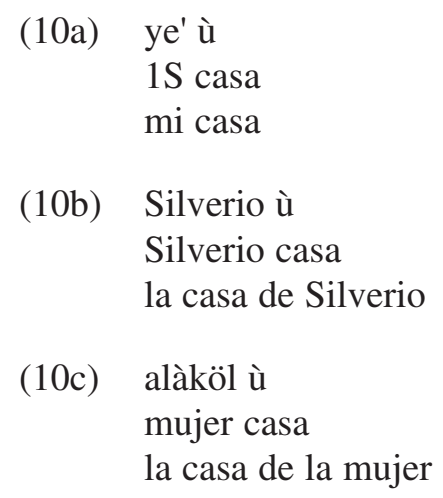

En el texto que aquí se incluye, encontramos muy pocos ejemplos de frases genitivas. Podemos señalar, sin embargo, que el nombre de la historia, Tcho' dawe, constituye una frase genitiva. A pesar de que los bribris llaman a estos personajes mitológicos diablos del bosque en español, su nombre significa literalmente "enfermedad del guacal", ya que se compone de tch $\underline{o}^{\prime}$ ( $t k \underline{a}^{\prime}$ en otros dialectos) 'guacal' y dawè 'enfermedad'. Otra frase genitiva en el texto se encuentra en la cláusula 33: di' ula ( $d i^{\prime}$ 'agua' ula 'brazo') 'brazo de río, riachuelo tributario de un río mayor'.

\subsection{Posposiciones}

El bribri es una lengua exclusivamente posposicional. Ejemplos de posposiciones como marcadores de casos que aparecen en el texto adjunto, son:

(11) Ergativo: Sibö tö íyök yawè.

Sibö ERG tierra hacer.IM

Sibö hizo la tierra.

(12) Dativo: $E^{\prime}$ mèke sa tö a' ia.

DEM dar.IM 1P ERG 2P DAT

Les daremos eso a ustedes.

(13) Experiente: Ie' ki i kiàne.

3S EXP $3 S$ quererse.PE

Él lo quería.

(14) Locativo: $\mathrm{E}^{\prime}$ bùkàmi di' a.

DEM lanzar.PE río en

Los lanzó al río 


$$
\begin{array}{lll}
\text { Instrumental: } & \text { Ie'pa s tchèkèlur ikè wa. } \\
& \text { 3P 1P punzar.IM.PL lanza } & \text { con } \\
& \text { Ellos nos punzaron con lanzas. }
\end{array}
$$

\subsection{Sustantivo-adjetivo}

El bribri casi sistemáticamente coloca el adjetivo después del sustantivo al que modifica. Ejemplos del texto son: ák wöchaka 'piedra pura', uséköl èbale 'sacerdote primero' (primer sacerdote), kapá taì 'jefe grande' (gran jefe), kó tạie 'lugar grande.INT' (lugar muy grande). En el texto hay una excepción que probablemente confirma la regla: es la muy marcada cláusula 50, una oración exclamativa atributiva: Tcho'dawe sulù pë' (Tcho'dawe malvada gente) '¡Los Tcho'dawe eran gente muy malvada!', donde el cambio de orden parece marcar énfasis en el modificador.

En algunos casos, lo que parece ser un modificador de sustantivo aparece detrás del verbo, de manera que la FN parece escindida. Estas FNs escindidas se deben al hecho de que en bribri los adjetivos y los adverbios no constituyen clases distintas de palabras (Constenla Umaña et al. 1998: 4), como puede observarse en los siguientes ejemplos:
(16a) alàköl taì
mujer grande 'una mujer grande'
(16b) ie' tsö taì
3S canta mucho 'ella canta mucho.'
(16c) alàköl bua'
mujer buena 'una mujer buena '
(16d) ie' tsö bua'
3S canta bien 'ella canta bien.'

La FN escindida comúnmente tiene que ver con modificadores cuantitativos, incluyendo numerales, como se ve infra. Ejemplos del texto son (cláusulas DC3-DC4):
Míshka sa di'ula síwèke
taì, datsi' pèke
taì.
ir.HORT 1P río-brazo secar.IM mucho, mataste cortar.IM mucho
Vamos a secar muchos ríos, a cortar mucho mataste (corteza para vestir).

Un ejemplo de corpora adicionales:

$\begin{array}{lllll}\mathbf{E}^{\prime} \quad \text { ishtàwe } & \text { i } & \text { di } & \text { kós } \\ \text { DEM contar.IM } & \text { 3S } & \text { ERG } & \text { todo } \\ \text { 'Ella contaba todo eso.' } & & & \end{array}$


Los numerales aparecen algunas veces después del sustantivo pero más frecuentemente después del verbo de la cláusula, como muestra el cuantitativo kós en la cláusula (18) supra; ejemplos del texto son (cláusulas 30 y 47):

$\begin{array}{llllll}\text { Kè̀këpa } & \text { siòdala } & \text { shkóke } & \text { ie'pa } & \text { ta } & \text { tchè̀l. } \\ \text { señor.PL } & \text { pobre.DIM } & \text { caminar.IM } & 3 \mathrm{P} & \text { COM } & \text { cuatro }\end{array}$

Los cuatro pobres señores caminaron con ellos.

\begin{tabular}{lllll} 
Pë' & batàla & tso' & ia & mañáál. \\
persona & resto & ST & todavía & tres \\
& \multicolumn{2}{l}{ Todavía quedaban tres de estas personas. }
\end{tabular}

De corpora adicionales:

$\begin{array}{llclll}\text { Ie' } & \text { léköli } & \text { ñe' } & \mathrm{e}^{\prime} & \text { wíköl yawè } & \text { èk. } \\ \text { 3S } & \text { mujer.ENF } & \text { que } & \text { DEM } & \text { imagen hacer.IMPF } & \text { una } \\ \text { 'De esa mujer él hizo una imagen.' } & \end{array}$

Sin embargo, los modificadores cuantitativos también pueden aparecer delante del sustantivo:

Welè $\quad$ yókòli $\quad$ yör $\quad$ tòtóla
algunos listos aprender.IM fácil.DIM
Algunos listos aprenden fácilmente.

Constenla Umaña et al. (1998: 5) afirman que cuando se usan como modificadores del sustantivo, los demostrativos se colocan después del sustantivo. Este es generalmente el caso (ver cláusulas 36, DC13 y 61 en el texto); sin embargo, en mis datos he encontrado ejemplos de demostrativos que aparecen delante del sustantivo, tales como:

$$
\begin{aligned}
& \tilde{\tilde{n} \underline{e}} \text { ák taì } \\
& \text { DEM piedra grande } \\
& \text { 'aquella gran piedra' }
\end{aligned}
$$

El siguiente ejemplo del texto (claúsula 25) muestra un demostrativo delante del sustantivo al que determina, pero parece haber también relativización, lo cual puede estar interviniendo en el ordenamiento de los elementos:

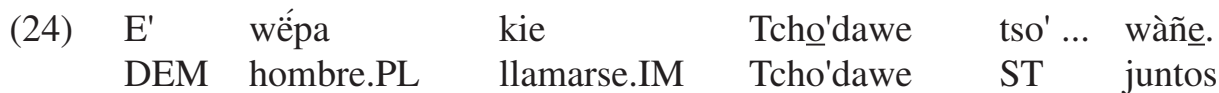
Esos hombres llamados Tcho'dawe estaban juntos...

El demostrativo puede funcionar como un marcador enfático o topicalizador. En tal caso siempre sigue al sustantivo: 


\begin{tabular}{|c|c|c|}
\hline I & $\begin{array}{l}\text { yë́ } \\
\text { DFM }\end{array}$ & $\begin{array}{lc}\mathrm{e}^{\prime} & \text { kie } \\
\end{array}$ \\
\hline & & \\
\hline
\end{tabular}

\subsection{Cláusula relativa}

Wilson (1984) aplica al bribri la jerarquía de relativización de FNs propuesta por Edward Keenan y Bernard Comrie's (1977). Según su estudio, excepto por el objeto de comparación, el bribri admite la relativización de cualquier FN. Concluye que

\footnotetext{
Bribri has a favorite strategy for relative clause formation which permits a variety of options. In all cases the NP which contains the head in the main clause is fronted, and the coreferential NP in the relative clause may optionally be fronted (p. 181).
}

La relativización no es un tema fácil en bribri; una caracterización exhaustiva rebasaría el objeto de este trabajo. Sin embargo, para no excluir el tema del todo, señalaré dos ejemplos del texto adjunto que muestran la estrategia preferida del bribri en cuanto a la relativización.

\section{Cláusula 2:}
$\mathrm{E}^{\prime} \quad$ tso
awène- ie'
tö, se' ditsö,
$\mathrm{e}^{\prime}$
rö Kóbala. DEM primero crear.IM 3S ERG 1P semilla DEM COP Kóbala Los primeros seres que él creo fueron los Kóbala.

De acuerdo con la descripción de la cláusula relativa que hace Payne (1997), en este ejemplo el núcleo de la construcción es el segundo demostrativo $e^{\prime}$. La cláusula restrictiva que modifica al núcleo, $e^{\prime}$ tsó awène $i e^{\prime}$ tö, aparece antepuesta y es seguida por una aposición explicativa, $s e^{\prime}$ ditsö, que es literalmente 'nuestra semilla' pero se refiere en este contexto a los seres humanos en general. La FN relativizada dentro de la cláusula restrictiva es $e^{\prime}$ ts $\underline{o^{\prime}}$. Una traducción más literal de la oración sería: 'Los primeros que él creó, (en cuanto a) seres humanos, esos fueron los Kóbala.'

Otro ejemplo del texto es el siguiente (cláusula 34):

$$
\begin{aligned}
& \text { Kòkekuö kàlkuö kièke kòkekuö èse pè shurù, } \\
& \text { mastate árbol llamarse.IM mastate } \\
& \text { El mastate del árbol llamado kòkekuö, ellos cortaron mucho de eso, }
\end{aligned}
$$

Aquí la FN relativizada dentro de la cláusula restrictiva es kòkekuö kàlkuö, y la FN nuclear dentro de la cláusula principal es el pronombre comparativo èse. El pronombre se compone del demostrativo $e^{\prime}$ y la partícula comparativa -se. Una traducción más literal de este ejemplo sería: 'El árbol de kòkekuö, llamado kòkekuö , ellos cortaron un montón de uno como ese.' 
A partir de estos dos ejemplos, podemos decir que el bribri prefiere el tipo prenominal de cláusula relativa, es decir, el orden: cláusula relativa-sustantivo.

\subsection{Verbo - Adverbio}

Muchos adverbios se colocan después del verbo, en particular aquellos que, como ya se mencionó, comparten la misma clase de palabras que los adjetivos. Aquí vemos algunos ejemplos del texto (cláusulas 21, 45, 39):

(28) Katöwạ séraë.

comer.IMP completamente

Cóma(lo) completamente.

(29) Dàmi sulù i yàmịpa éna

llegar.IM mal $3 \mathrm{~S}$ pariente.PL EXP

Sus parientes lo tomaron mal.

(30) Pàterèwa bua'ë kòkekuö wa.

envolver.IM bien.INT mastate INST

(Ellos lo) envolvieron muy bien con el mastate.

Los adverbios locativos también son posverbales (ver cláusulas 51, 52 y 53). Sin embargo, los elementos adverbiales que se refieren al tiempo tienden a ser iniciales de cláusula (cláusula 40, ver también cláusulas 1, 26, 58 y 69):

(31) Bulàmi ie' shkène, mañana 3S despertar.IM

Por la mañana él se despertó,

Esto es perfectamente natural, ya que, mientras que adverbios como bien, mal y completamente tienen un alcance semántico limitado al verbo, los adverbios de tiempo tienden a tener un alcance semántico que abarca la cláusula como un todo (cp. Givón 1984: 81-2). Por la misma razón, adverbios de comentario como bö rö 'talvez' (cláusula DC8) y e'ñerma 'realmente' (cláusula 44) aparecen al principio de sus respectivas cláusulas. Este hecho claramente muestra que, en cuanto al OP de verbo y adverbio, no es recomendable tratar los adverbios como una clase de elementos unificada, como parece sugerir la lista de parámetros de Vennemann. 


\subsection{Verbo - Auxiliar}

En cuanto a los auxiliares, es aplicable el mismo comentario hecho en la sección anterior en relación con los adverbios. No todos los auxiliares se comportan necesariamente del mismo modo en cuanto a OP; el bribri presenta un ejemplo interesante en este sentido. Las perífrasis verbales que expresan significado progresivo y final son de orden auxiliar-verbo y en ellas el auxiliar se flexiona mientras que el verbo principal puede aparecer en su forma no finita (marcada por los sufijos - ök $o$ - $\underline{u} k$ ) o bien flexionado; en los demás casos las perífrasis son de orden verbo-auxiliar y el verbo principal aparece siempre flexionado. He aquí algunos ejemplos (cláusula 12):

(32) Tchó'dawe bák ie'pa ñìwábalök sulùë,

Tcho'dawe ST.PE 3P dañar.INF mal.INT

Los Tcho'dawe estuvieron haciendo mucho daño a la gente

Este ejemplo es especialmente ilustrativo porque muestra el verbo auxiliar flexionado $b a ́ k$, forma perfectiva (y supletiva) del verbo estativo/existencial tso'. El siguiente ejemplo (cláusula 56) muestra un verbo semánticamente más cargado como auxiliar, flexionado y seguido por la forma no finita del verbo principal:

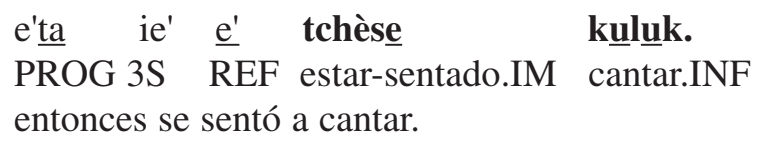

Además del verbo estativo/existential $t s o^{\prime}$, que sirve como auxiliar en las perífrasis progresivas, existe en bribri un conjunto de formas llamadas por Constenla Umaña et al. (1998: 67) posicionales y que yo prefiero llamar "auxiliares de posición". Estos auxiliares sirven como verbos estativos/existenciales, pero al mismo tiempo especifican la posición en que se encuentra la entidad de la cual se habla. Estos auxiliares se colocan más frecuentemente después del verbo, como en los siguientes ejemplos del texto (cláusulas 34-35 y 68):

$\begin{array}{lllll}\text { èse } & \text { pè } & \text { shurù, } & \text { tulè } & \text { arkị. } \\ \text { DEM.COMP } & \text { cortar.IM } & \text { mucho } & \text { colgar.IM } & \text { AUX } \\ \text { ellos cortaron un montón de eso y lo dejaron colgado. } & \end{array}$

i $\quad$ ñìpöke tulur di' wö́chika;
3S lanzar.IM AUX agua pura
ellos estaban lanzándose al agua;

En corpora adicionales he encontrado múltiples ejemplos de esta colocación del auxiliar de posición después del verbo principal:

$$
\begin{aligned}
& \text { Bikili' démi ar } \text { ar bó tsóka. } \\
& \text { zorro ir.PE AUX fuego sobre } \\
& \text { El zorro fue a colgarse sobre el fuego. }
\end{aligned}
$$


(37)

Sorbulu e'bale tulur...

Sorbulu esconder.IM AUX

Los Sorbulu estaban escondidos...

(38) I muè tcher...

3S amarrar.IM AUX

Él estaba amarrado...

(39) Tsanè démi ar dikö ki.

pájaro-carpintero llegar.PE AUX pejibaye sobre

El pájaro carpintero fue a pegarse del palo de pejibaye.

En todos estos casos, el verbo principal está flexionado (los auxiliares de posición nunca se flexionan). Cuando se da el orden inverso, es decir, verbo-auxiliar, el verbo principal se emplea en su forma no finita:

(40) E' kuék këkëpa tuluạt se' yuwök. DEM por mayor.PL AUX 1P hacer.INF

Por esto (es que) los mayores continúan haciéndonos ${ }^{7}$.

\subsection{Verbo -modal}

Un modal es una forma verbal que codifica la actitud sicológica del hablante con respecto a la acción de la que habla: quiero/debo/puedo comer. En bribri, algunos de estos significados se expresan léxicamente, por ejemplo, 'querer' se expresa por medio del verbo de voz media kiànuk 'necesitarse' (ver cláusula 5); la obligación se expresa mediante la palabra modal káwö̈ta , que no presenta flexión de tiempo/aspecto (pero tampoco se flexiona el verbo principal que la sigue); además, otros significados modales se expresan mediante sufijos verbales o flexión. Por estas razones, tampoco es viable, de nuevo frente al modelo de Vennemann, examinar esta correlación como un parámetro independiente para la tipología de OP.

En el texto incluido, se puede señalar un ejemplo (cláusula 26) que muestra un tipo de construcción modal-verbo (ambas formas aparecen flexionadas):

(41) E' kếpa ñèe ie'pa wồkitchồka mìa cha uséköl e' èbale DEM después PROG 3P decidir.IM ir.IM PROG sacerdote DEM primero Entonces ellos decidieron ir al primer sacerdote 


\section{Conclusión}

En relación con el modelo de Vennemann, el OP del bribri no sería consistente porque mientras que en algunos parámetros se muestra como una lengua adjunto-núcleo, en otros tiende a ser núcleo-adjunto. Sin embargo, los parámetros en que el bribri muestra OP rígido, son todos del mismo tipo: adjunto-núcleo. Este es el caso para los siguientes parámetros:

1. Objeto - Verbo

2. Genitivo - Sustantivo

3. Sustantivo - Posposición

Otros parámetros muestran como orden básico el tipo núcleo-adjunto, pero en esos casos el OP no es tan rígido como lo es en cuanto a los parámetros mencionados: los adjetivos/adverbios tienden a ser desplazados al final de la cláusula; los demostrativos algunas veces aparecen delante del sustantivo; las perífrasis verbales pueden ser Aux-V o V-Aux; en las FNs encontramos fundamentalmente el orden sustantivo-adjetivo pero las cláusulas relativas son típicamente prenominales.

Dados estos hechos, el bribri se puede caracterizar, en cuanto a tipología de OP, como una lengua altamente consistente con el tipo (d) (ver 2.1 supra: SOV, Posp, genitivo-sustantivo, sustantivo-adjetivo). Esta caracterización apoya el conjunto de parámetros propuesto por Greenberg, en oposición al modelo propuesto por Vennemann. Como se ha visto, los parámetros en los que es problemático ajustarse al modelo de Vennemann son, precisamente, aquellos que él apunta como correlaciones separadas, es decir: verbo-adverbio, verbo-auxiliar/modal y sustantivo-cláusula relativa.

En comparación con otras lenguas chibchas, vale la pena notar que los hechos acerca del OP bribri son muy semejantes a los que presenta Craig (1986) acerca de la lengua rama. Esto sugiere opciones muy interesantes para explorar en relación con la sintaxis diacrónica de OP en la familia chibcha. De hecho, la muestra de Greenberg incluye la lengua chibcha/muisca de Colombia, la cual describe como SOV, posposicional, sustantivo-adjetivo, demostrativo-sustantivo y sustantivo-numeral. Básicamente, esta caracterización sirve igualmente para el bribri (excepto, como vimos, en la correlación demostrativo-sustantivo), lo cual muestra que los rasgos tipológicos de OP del protochibcha podrían coincidir básicamente con los del chibcha, el bribri y el rama.

Finalmente, puede ser útil señalar aquí algunos de los problemas que pueden complicar el estudio de OP en cualquier lengua y que, de ser considerados, podrían afectar notablemente hechos tales como los que se han presentado aquí con respecto a la lengua bribri:

1. Los estudios de OP se basan siempre en FNs plenas. El estatus de los pronombres en relación con los parámetros relevantes usualmente no se considera porque a menudo ellos se comportan de manera diferente, y esto podría distorsionar los hechos establecidos con respecto al OP. 
2. Las oraciones básicas (es decir, no marcadas) del discurso a menudo no presentan FNs plenas que codifiquen sujeto y objeto; frecuentemente una o ambas funciones se eliden debido a su recuperabilidad del contexto previo. Esta situación hace más difícil el establecimiento del OP en términos de discurso continuo.

3. Se ha afirmado (Mallinson \& Blake 1981) que solo el $40 \%$ de las lenguas del mundo se ajustan al modelo de adjunto y núcleo. Esto representa un problema serio para aquellos que defienden una regla universal de estructura fraseal según la cual los adjuntos consistentemente preceden o siguen al núcleo al que se refieren.

\section{Notas}

1. Los térrabas, un grupo cercanamente relacionado con los téribes de Panamá, y los guaymíes (tanto moveres como bocotás) emigraron a Costa Rica desde Panamá.

2. Una descripción detallada acerca de los rasgos lingüísticos propios del área se encuentra en Constenla Umaña (1991).

3. La palabra Talamanca no es de origen indígena. Aparece por primera vez en documentos de 1605 del español Diego de Sojo, quien bautizó el valle de acuerdo con el nombre de su propio lugar de nacimiento en España (Stone 1961: 12; Guevara y Chacón 1992: 31).

4. Esta afirmación se basa en el estudio sobre las tres variantes dialectales que me encuentro realizando actualmente.

5. Los términos utilizados por Venneman son Operand $=$ Núcleo y Operator $=$ Adjunto.

6. Las cláusulas correspondientes a discurso citado (DC) no fueron consideradas en este conteo.

7. Esta oración se refiere a la creencia bribri de que los seres encargados de crear a los seres humanos, los sula', continúan creándolos a lo largo de la vida de la persona.

\section{Bibliografía}

Anderson J. and C. Jones (eds.). 1974. Historical Linguistics. Amsterdam: North-Holland.

Bozzoli, Ma. Eugenia. 1975a. Localidades indígenas costarricenses. San José: Educa.

1975b. Birth and death in the belief system of the Bribri Indians of Costa Rica. Ph. D. dissertation. University of Georgia.

1979. El nacimiento y la muerte entre los bribris. San José: Editorial de la Universidad de Costa Rica. 
Campbell, Lyle, Leslie Saxon and Vit Bubenik. 1988. "Word order universals: Refinements and clarifications", Canadian Journal of Linguistics. 33(3): 1-22.

Comrie, Bernard. 1989. Language universals and linguistic typology. Chicago: The University of Chicago Press.

Constenla Umaña, Adolfo. 1985. "Clasificación lexicoestadística de las lenguas de la familia chibcha". Estudios de Lingüística Chibcha. 4: 155-97.

1989. "La subagrupación de las lenguas chibchas: algunos nuevos indicios comparativos y lexicoestadísticos". Estudios de Lingüística Chibcha. 8: 17-97.

1991. Las lenguas del Área Intermedia: Introducción a su estudio areal. San José: Editorial de la Universidad de Costa Rica.

Constenla Umaña, Adolfo, Feliciano Elizondo Figueroa y Francisco Pereira Mora. 1998. Curso básico de bribri. San José: Editorial de la Universidad de Costa Rica.

Craig, Colette G. 1986. "The Rama language: a text with grammatical notes". Estudios de Lingüistica Chibcha. 5: 21-44.

Gabb, William. 1875. "On the Indian tribes and languages of Costa Rica". Proceedings of the American Philosophical Society. 14: 483-602.

Givon, Talmy. 1984. Syntax. A functional-typological approach. Vol I. Amsterdam: John Benjamins.

Guevara, Marcos y Rubén Chacón. 1992. Territorios indios en Costa Rica. San José: García Hermanos.

Greenberg, Joseph. 1966. "Some universals of grammar with particular reference to the order of meaninful elements." En Greenberg, J. (ed.) Universals of Language. Cambridge: M.I.T. Press.

Hawkins, John. 1983. Word order universals. New York: Academic Press.

Jara, Carla Victoria. 1993. Historias bribris. San José: Editorial de la Universidad de Costa Rica.

Jara, Carla Victoria y Alí García Segura (en prensa). Diccionario de Mitología Bribri. San José: Editorial de la Universidad de Costa Rica.

Keenan, Edward and Bernard Comrie. 1977. "Noun phrase accessibility and universal grammar". Linguistic Inquiry. 8: 63-99. 
Mallinson, Graham and Barry J. Blake. 1981. Language typology. Amsterdam: North-Holland.

Margery, Enrique. 1982. Diccionario fraseológico bribri-español español-bribri. San José: Editorial de la Universidad de Costa Rica.

Payne, Thomas E. 1997. Describing morphosyntax. Cambridge: Cambridge University Press.

Pittier, Henry. 1898. Die Sprache der Bribri Indianer in Costa Rica. Sitzungsberichte der kais. Win der Wiss. Ed. 138.

1903. "Folk-lore of the Bribri and Brunca Indians of Costa Rica". Journal of American Folk-lore. 16 (60): 1-9.

Stone, Doris. 1961. Las tribus talamanqueñas de Costa Rica. San José: Museo Nacional.

Schlabach, Raymond. 1974. "Los fonemas del bribri del Valle de Talamanca". América Indígena. 34 (2): 355-62.

Venneman, Theo. 1974. "Topics, subjects and word order: From SXV to SVX via TVX". En Anderson J. and C. Jones (eds.).

Wilson, Jack. 1982. "El alfabeto bribri". En Margery 1982.

1984. "Relative clauses in Bribri". Estudios de Lingüística Chibcha. 3: 179-99.

\begin{tabular}{|c|c|c|c|}
\hline \multicolumn{4}{|l|}{ Abreviaturas } \\
\hline $1 \mathrm{~S}$ & primera persona singular & ERG & ergativo \\
\hline $2 \mathrm{~S}$ & segunda persona singular & EXP & experiente \\
\hline $3 \mathrm{~S}$ & trercera persona singular & HORT & hortativo \\
\hline $1 \mathrm{P}$ & primera persona plural & IM & imperfectivo \\
\hline $2 \mathrm{P}$ & segunda persona plural & IMP & imperativo \\
\hline $3 \mathrm{P}$ & tercera persona plural & INF & infinitivo \\
\hline AG & agentivo & INST & instrumental \\
\hline AUX & auxiliar & INT & intensificador \\
\hline $\mathrm{COM}$ & comitativo & INTR & interjección \\
\hline COMP & comparativo & LOC & locativo \\
\hline CONN & conector & NEG & negación \\
\hline $\mathrm{COP}$ & cópula & PE & perfectivo \\
\hline DAT & dativo & PL & plural \\
\hline DEM & demostrativo & PROG & progresivo \\
\hline DIM & diminutivo & REF & reflexivo \\
\hline EFF & efecto & ST & estativo \\
\hline ENF & enfático & VOC & vocativo \\
\hline
\end{tabular}




\section{ANEXO}

\section{Tcho'dawe 'Los diablos del bosque'}

1. E' kë́pata Sibö tö íyök yawè.

DEM después dios ERG tierra hacer.IM

Después de eso Sibö̀ (dios) hizo la tierra.

2. E' tsọ' awène ie' tö, se' ditsồ, e' rö Kóbala. DEM primero crear.IM 3S ERG 1P semilla DEM COP Kóbala Los primeros seres que hizo fueron los Kóbala.

3. Lồneka taì, multiplicarse.IM mucho

Se multiplicaron mucho

4. êma erë ie' i mowène.

PROG CON 3S 3P tratar.IM

pero entonces él los probó.

5. Ie' kị i kiàne i mòsopa iạ katànok, yànok, 3S EXP 3P necesitarse.PE 3S ayudante.PL DAT comer-para tomar-para Los quería como comida y bebida para sus ayudantes,

6. kề i di bua',

NEG 3P COP bueno

pero no eran buenos,

7. ák wö̀chaka è $\mathrm{i}$ di. piedra pura solamente 3P COP eran pura piedra.

8. E' kuék ie' tö e' èwewạ, EF $3 S$ ERG DEM eliminar.IM Por eso los eliminó

9. $\underline{\text { ema }}$ e' bùkàmi di' a . PROG DEM lanzar.PE agua LOC lanzándolos al agua.

10. E' skè tsakìne i di: DEM otro nacer.PE 3S ERG Entonces creó otros,

11. e' rö Tcho'dawe.

DEM COP Tcho'dawe esos fueron los Tcho'dawe. 
12. Tchó'dawe bák ie'pa ñìwábalök sulùë, Tchódawe ST.PE 3P daño.INF mal.INT Los Tcho'dawe estaban haciendo mucho daño,

13. ie'pa $\mathrm{s}$ tèkèlur, 3P 1P matar.IM.PL mataban a la gente,

14. ie'pa s tchèkèlur ikè wa. 3P 1P punzar.IM.PL lanza INST la punzaban con lanzas.

15. Be' mìa ie' a di'ula kiök, $2 \mathrm{~S}$ ir.IM 3P DAT río-brazo preguntar.INF Si usted va a pedirles un bracito de río

16. siúwa po è; secar.IMP seco solamente séquelo completamente;

17. e' a yawì tso', i tso', DEM LOC cangrejo ST 3S ST si hay cangrejos,

18. pulè tso', i tso, caracol ST 3S ST si hay caracoles,

19. kachötak tso', babosa ST si hay babosas,

20. e' tsúbitu, tsúbitu DEM traer.IMP traer.IMP tráigalos, tráigalos

21. katöwa séraë, comer.IMP completamente y cómaselos completamente,

22. e'ta kè̀ ba ttèwa i tö. PROG NEG 2S matar.IM 3S ERG para que esas cosas no lo maten.

23. $\mathrm{Be}^{\prime} \mathrm{r} \quad \mathrm{e}^{\prime}$ mèat, 2S ERG DEM dejar.IM

Pues si usted los deja, 
24. be' ttèwa i di.

2S matar.IM 3S ERG

ellas te matarán.

25. E' wëpa kie Tcho'dawe tso' kó wịki kó wàñe. DEM hombre.PL llamarse.IM Tcho'dawe ST lugar LOC lugar juntos Esos hombres llamados Tcho'dawe estaban juntos en un lugar allá arriba.

26. E' këpa ñèe ie'pa wökitchöka mìa cha uséköl e' èbale DEM después PROG 3P decidir.IM ir.IM PROG sacerdote DEM primero Después de eso decidieron ir a donde los primeros sacerdotes

27. (tso' tche kapá taì, awá)

ST ya jefe grande médico

(para entonces ya había grandes jefes y médicos)

26. e' kiök aìe.

DEM invitar.INF allá-arriba

para invitarlos allá arriba..

28. Ie'pa mìa uséköl ska, 3P ir.IM sacerdote LOC

Fueron al lugar de los sacerdotes

29. töraạ:

decir.IM

y dijeron:

DC1. "Míshka, a naùpa, míshka, a naùpa, ir.HORT VOC tío.PL ir.HORT VOC tío.PL

"Vamos, oh tíos, vamos,

DC2. sa ska Anéu wìe,

1P LOC Anéu allá-arriba

a nuestro lugar, Anéu, allá arriba,

DC3. míshka sa di'ula síwèke taì, ir.HORT 1P río-brazo secar.IM mucho vamos a secar nuestros ríos,

DC4. datsi' pèke taì, mastate cortar.IM mucho a cortar un montón de (corteza) de mastate, 
DC5. e' mèke sa tö a' ia.." DEM dar.IM 1P ERG 2P DAT

les daremos todas esas cosas a ustedes."

30. Këkëpa siòdala shköke ie'pa tạ tchél. señor.PL pobre.DIM caminar.IM 3P COM cuatro

Los pobres señores, cuatro de ellos, se fueron con los Tcho'dawe.

31. Uséköl bitè , sacerdote venir.IM

Los sacerdotes vinieron,

32. i kalépa dàtse taiëe Anéu kicha. 3S ayudante.PL llegar.IM mucho.INT Anéu LOC muchos de sus ayudantes vinieron a Anéu.

33. Ie'pa tso' ie'pa ttöke di'ula síwèke, ì wè̀ke... 3P ST 3P hablar.IM río-brazo secar.IM qué hacer.IM Ellos estaban hablando de secar ríos, de hacer cosas...

34. Kòkekuö kàlkuö kièke kòkekuö mastate árbol llamarse.IM mastate La corteza (mataste) del árbol llamado kòkekuö

34. èse pè shurù, DEM.COMP cortar.IM mucho cortaron un montón de eso

35. tulè arkị. colgar.IM AUX y lo colgaron.

36. Kó $\quad e^{\prime}$ nañéwe tạ, ie' wạ kapá taì táwa tiempo DEM noche entonces 3S AG jefe grande matar.PE Esa noche uno de ellos (Tcho'dawe) decide matar a un jefe.

37. Kalö̀wewa, agarrar.IM Lo agarró,

38. ttèlur, matar.IM lo mató,

39. pàterèwa bua'ë kòkekuö wa. envolver.IM bien.INT mastate INST lo envolvió muy bien con el mastate. 
40. Bulàmi ie' shkène, mañana $3 \mathrm{~S}$ despertar.IM

A la mañana siguiente se despertó

41. chè cha:

decir.IM PROG

y dijo:

DC6. "Shkène, shkènẹ!" despertar.IM despertar.IM

"¡Despierte, despierte!"

42. Këkëpala kề shkène. señor.DIM NEG despertar.IM

El señor no se despertó.

43. Ie' töraa:

3S decir.IM

Dice:

DC7. "Alàr, i saú, niños $3 \mathrm{~S}$ ver.IMP

"Niños, mírenlo,

DC8. börö su bunàne tal vez $1 \mathrm{P}$ caer.PE talvez $1 \mathrm{~S}$ morir.IM tal vez se cayó y se murió!"

44. E'ñerma ie' i ttèwa. en-realidad 3S 3S matar.IM INT

En realidad él lo había matado. Ahhh!

45. Dàmi sulù i yàmịpa éna llegar.IM mal 3S pariente.PL EXP Su familia tomó esto muy a mal.

46. I che:

3S decir.IM

Dijeron:

DC9. "I núbalö!" 3S envolver.IMP

"Envuélvanlo!" 
47. Pë' batàla tso' ia mañál, persona resto ST todavía tres Todavía quedaban tres de ellos

48. e' rö kapà.

DEM COP jefe

que eran jefes.

49. E' tö i che:

DEM ERG 3S decir.IM

Ellos dijeron:

DC10. "Sa' mìyaltche."

1P irse-ya.IM

"Ya nos vamos."

DC11. "Èkë, a' mukk mìkela,

INTR 2P acompañar.IM ir.IM.DIM

"Muy bien, los acompañamos,

DC12. s ò̀te siòrë, siòrë."

1P quedarse.IM triste.INT triste.INT

pero nos quedamos muy tristes, muy tristes."

50. Tcho'dawe sulù pë'!

Tcho'dawe malo persona

¡Los Tcho'dawe son gente mala!

51. I yàmịpa kalöwewa dià $\mathrm{i}$ di, 3P pariente.PL agarrar.IM allá-abajo 3P ERG

Agarraron a sus familias de allá abajo,

52. mìchòne aìe.

regresar.IM allá-arriba

y regresaron allá arriba.

53. E' dè̀kane aìe,

DEM llegar.PE allá-arriba

Cuando llegaron allá arriba,

54. e'ta i chè i di:

PROG 3s decir.IM 3S ERG

uno de ellos dijo:

DC13. "Tchọ'dawe e' tö cha su wéibitu kó mịkạa;

Tcho'dawe DEM ERG PROG 1P molestar.IM tiempo TIME

"Esos Tcho'dawe nos han estado molestando por mucho tiempo; 
DC14. e' kuéki ìñe $s$ déútu tèwa $i$ di, EF hoy 1P difunto matar.IM 3S ERG por eso ahora que han matado a uno de los nuestros,

DC15. ìñe yö i skè kachèkène." hoy 1 S.ERG $3 \mathrm{~S}$ otra vengarse.IM yo me vengaré de ellos."

55. Ñèe ie' dèmine $\underline{e}$ aì, PROG 3S regresar.IM allá-arriba Entonces cuando regresó allá arriba,

56. e'ta ie' ê' tchèse kụluk, kóli kiök:

PROG 3S REF sentarse.IM cantar.INF lluvia llamar.INF se sentó a cantar llamando a la lluvia:

DC16. "Kóli tsör, kóli tsör", lluvia caer.IM lluvia caer.IM "Que llueva, que llueva",

57. ie' kulè̀.

3S cantar.IM cantaba.

58. Kó tchél tạ, i yàmịpa a $\mathrm{i}$ che: día cuatro entonces $3 \mathrm{~S}$ pariente.PL DAT $3 \mathrm{~S}$ decir.IM Después de cuatro días él les dijo a sus parientes:

DC17. "A' bitsö!"

2P ayunar.IMP

"¡Ayunen!"

59. Ska dè kó tchél tạ, otro pasar.IM día cuatro entonces Otros cuatro días pasaron,

60. be' i sụwè kóli bitutsẹ, 2S $3 \mathrm{~S}$ ver.IM lluvia venir.IM entonces se ve la lluvia venir,

kóli bitutse,$\quad$ kóli bitutse lluvia venir.IM lluvia venir.IM y viene la lluvia, viene la lluvia... 
61. E'tạ kó i' ki ie'pa tso' PROG lugar DEM LOC 3P ST Entonces ese lugar donde ellos estaban

62. kó taîe di' bitutse cha lugar grande.INT agua venir.IM PROG ese enorme lugar donde cayó la lluvia

63. (ye' a tâ, èse kièke $\mathrm{a}^{\prime} \quad \mathrm{r}$ "dilùbio"), 1S DAT PROG COMP llamarse.IM 2P ERG diluvio (para mí eso es lo que ustedes llaman el diluvio),

64. uuuf, tchörö kótö aì suköt.

INT llegar.IM falda allá-arriba LOC

uhh, estaba lleno hasta las faldas de aquella montaña.

65. Tcho'dawe kalöwe di' tö, Tcho'dawe agarrar.IM agua ERG El agua agarró a los Tchódawe

66. uffft, dökạ.

INT cubrir.IM

y los cubrió.

67. Tchó'dawe tsồtchö sulùë.

Tcho'dawe bucear.IM mucho.INT

Los Tcho'dawe trataban de bucear a toda costa.

68. Aì kóbita Nomọööl, aìe èe, aquella montaña Nomòsöl allá arriba allí Allá arriba en la montaña Nomòsöl,

i ñìpö̀ke tulur di' wö̀chika;

3S lanzarse.IM AUX agua pura

se lanzaban a la pura agua;

69. bërë be' i sawe wịe kóbita tchër Muléröö, al-rato 2S 3S ver.IM allí montaña AUX Mulérök al rato usted los ve salir allá por la montaña Mulérök,

70. èe Tcho'dawe dàmika, allí Tcho'dawe salir.IM salían por allá 
71. erë ie' kö̀chö:

CONN 3P falso

pero era inútil:

72. ie' dawö̀a

3P morir.IM

se morían.

73. Ema ès Tchó'dawe éòwa Sibồ tö.

PROG COMP Tcho'dawe eliminar.IM Sibồ ERG

Así, de esta manera, Sibö eliminó a los Tcho'dawe.

74. Sulù ie' wàbalö

malo 3P hacer.IM

Hacían tanto daño,

75. e' kuék Kóbala e' éòwa Sibồ tö.

EFF Kóbala DEM eliminar.IM Sibö ERG

por eso Sibö eliminó a los Kóbala.

76. Kë rö bua' katànok;

NEG COP bueno comer-para

No eran buenos como comida,

77. kè rö bua'.

NEG COP bueno

no eran buenos.

78. $E^{\prime}$ ske kùnène

DEM otro crear.PE

Entonces creo a otros (seres),

79. $\mathrm{e}^{\prime}$ rö se', $\mathrm{e}^{\prime}$ rö $\mathrm{se}^{\prime}$,

DEM COP 1P DEM COP 1P

esos somos nosotros, esos somos nosotros.

80. ìke, be' éna i òne?

INT 2S EXP 3S caer.PE

ea, entiendes? 